\title{
Unsuspected colorectal carcinoma on routine abdominopelvic computed tomography
}

Su Ann Lee ${ }^{1}$, MBChB, FRCR, Angeline $\underline{P o h}^{1}$, MBBS, FRCR

\begin{abstract}
Colorectal carcinoma is a common lethal disease with signs and symptoms that may be nonspecific. Computed tomography (CT) of the abdomen and pelvis with or without contrast is frequently performed for various general abdominal complaints, but unlike CT colonography, the large bowel may not be optimally prepared for evaluation. As such, careful and diligent assessment of the non-prepared colon in all CT images of the abdomen and pelvis is important, as it ensures that incidental colorectal malignancy is not missed, especially in older patients. This article gives an overview of multidetector CT imaging signs and subtle clues to aid in the diagnosis of colorectal carcinoma, as well as their pitfalls.
\end{abstract}

Keywords: colorectal carcinoma, CT abdomen and pelvis, imaging findings, incidental, pitfalls

\section{INTRODUCTION}

Colorectal carcinoma $(\mathrm{CRC})$ is the third most common cancer in the world and the fourth most common cause of cancer deaths worldwide, affecting both genders almost equally. ${ }^{(1,2)}$ An annual global estimate of 1.2 million new cases and 600,000 deaths from CRC were recorded in 2008. ${ }^{(1-4)}$ The incidence and mortality rates for CRC increase with age; $90 \%$ of patients are aged 50 years or older. ${ }^{(1)}$ Significant geographical variation has been observed, with higher rates found in developed countries. ${ }^{(1-3)}$ Most CRCs develop from adenomatous polyps (adenoma-carcinoma sequence), which have a natural history of ten years. ${ }^{(5)}$ Patients with early CRC are often asymptomatic. The most common symptom is rectal bleeding. Other symptoms include changes in bowel habits, weight loss and abdominal pain.

Computed tomography colonography (CTC) has been validated as a screening and diagnostic tool for CRC in the United States, as it is comparable to optical colonoscopy in CRC detection. ${ }^{(4,6)}$ However, with the increase in non-CTC abdominopelvic CT studies being performed for other indications, CRC may also be incidentally detected in these images. It is therefore important for radiologists to be familiar with the imaging findings of CRCs and aware of the pitfalls of detecting CRC in non-CTC abdominopelvic CT studies, in order to avoid missing an unsuspected tumour.

\section{CTC VS. NON-CTC ABDOMINOPELVIC CT}

The key differences between CTC and non-CTC abdominopelvic $\mathrm{CT}$ are bowel preparation and colonic insufflation for bowel distension. ${ }^{(7)}$ To reduce the amount of faecal material within the colon, bowel preparation for CTC in our institution typically includes diet modification and oral or saline catharsis such as sodium biphosphate (bisacodyl) or polyethylene glycol-electrolyte solution. For example, faecal tagging with Tagitol is prescribed to assist in differentiating residual faeces and true polyps. Colonic distension is achieved with automated insufflation of carbon dioxide. Finally, volumetric multidetector $\mathrm{CT}$ is performed from the dome of the diaphragm to the symphysis pubis in prone and supine positions, with low osmolar intravenous (IV) contrast to further aid in the detection of polyps, with either a 64 or 320 multidetector CT scanner (Toshiba, Aquilion and Aquilion One). Adequate distension of the colon allows virtual colonoscopic assessment with 'fly through' and 'fillet views' using a specialised, postprocessing software (Vital Images, Vitrea).

In contrast, specialised bowel preparation, colonic insufflation and faecal tagging are not performed for non-CTC abdominopelvic CT. Rectal contrast is only used in cases of known rectal pathology, such as in CRC staging. The patient is only scanned in the supine position. The lack of bowel preparation and colon insufflation are the primary factors that contribute to the ease at which an unsuspected CRC may be missed or misinterpreted in evaluating such studies.

\section{IMAGING FINDINGS OF CRC ON NON-CTC ABDOMINOPELVIC CT}

The typical appearances of CRC on non-CTC abdominopelvic CT are short-segment eccentric bowel wall thickening and enhancement $^{(8)}$ or a discrete annular soft tissue mass causing luminal narrowing (Fig. 1). ${ }^{(8,9)}$ Primary CRC can also manifest as an intraluminal polypoidal mass or a large mass with central necrosis. ${ }^{(9)}$ However, in cases where the primary tumour is not obvious, recognising subtle secondary signs may help in detection. Focal pericolic neovascularity, fat stranding and locoregional adenopathy may be more conspicuous indicators of a tumour, although they may also be seen in other inflammatory bowel conditions such as diverticulitis and are therefore not specific. Bowel wall thickening proximal to an obstructing CRC has been shown to occur in $1 \%-7 \%$ of patients with partial or complete bowel obstruction, representing obstructive colitis that is postulated to be due to bowel wall ischaemia. ${ }^{(10)}$ The presence of multiple hypovascular masses in the liver that are suspicious for metastases should prompt a search for a primary tumour in the colon. Other sites of metastasis are the lungs, adrenals and bones. ${ }^{(9)}$ 

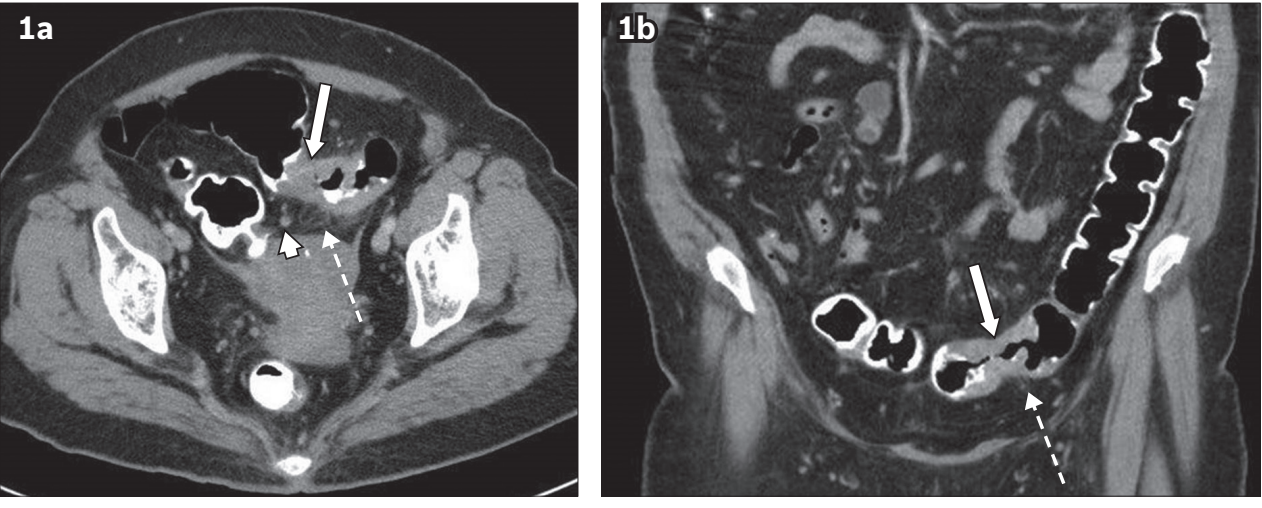

Fig. 1 (a) Axial and (b) coronal CTC images show the typical features of colorectal carcinoma: short segment annular wall thickening of the sigmoid colon (arrows) in an adequately distended large bowel, associated pericolic fat stranding (broken arrows) and enlarged lymph nodes (arrowhead).
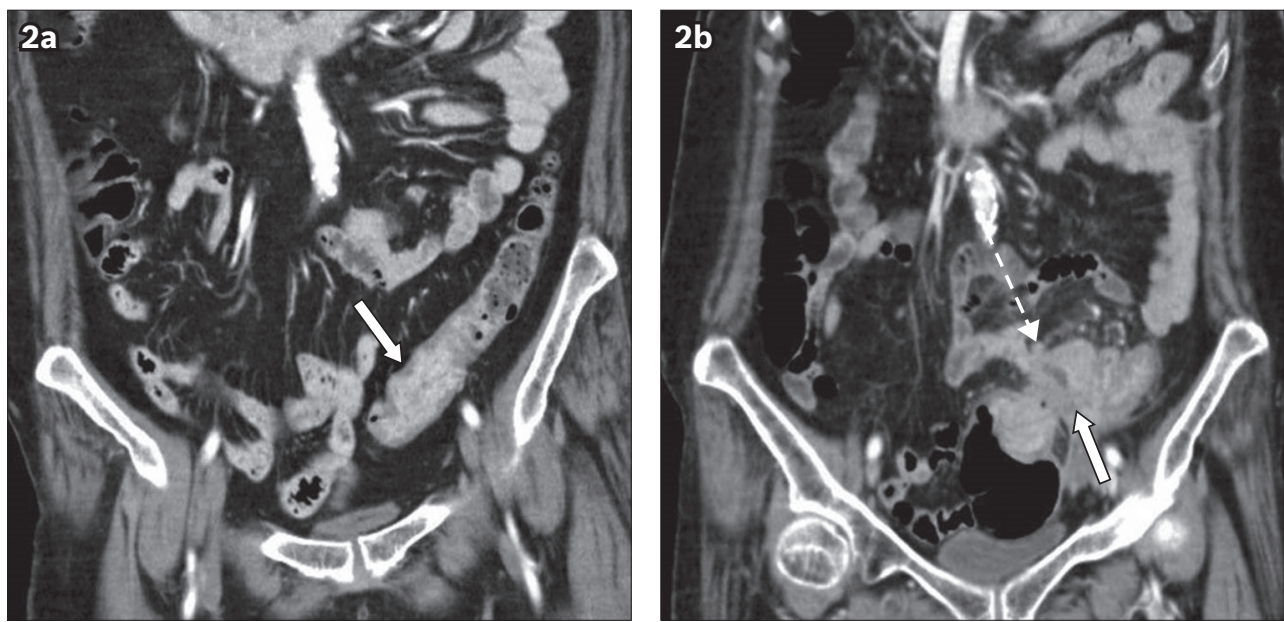

Fig. 2 Coronal CT images of a 59-year-old woman with gross haematuria show the difficulty in differentiating a collapsed bowel from a tumour. (a) There is focal enhancement and thickening of the sigmoid-descending colon junction (arrow), which was thought to be due to under-distension and apposition of normal enhancing mucosa. No pericolic abnormalities were noted at that time. (b) The patient presented with rectal bleeding two years later. There is now a heterogeneous enhancing mass (arrow) with pericolic fat stranding and enlarged loco-regional nodes (broken arrow).

\section{DIFFERENTIAL DIAGNOSIS OF CRC}

Other conditions such as diverticulitis and inflammatory bowel disease may present with similar imaging findings as CRC. ${ }^{(11)}$ These include bowel wall thickening, pericolic fat stranding and locoregional lymph nodes. There are, however, some differentiating features such as the pattern and extent of bowel wall thickening and degree of pericolic fat stranding in relation to the bowel wall thickening. ${ }^{(12-14)}$ The presence of diverticula, stratified mural enhancement and disproportionate pericolic and mesenteric fat stranding in relation to the bowel wall thickening, as well as more segmental involvement as opposed to focal involvement, tend to favour diverticulitis. In inflammatory bowel disease such as Crohn's disease, differentiating features include segmental bowel wall thickening, 'skip lesions', perivascular engorgement, fistulation and fibrofatty proliferation. Acute infective colitis in CRC also tends to have more segmental as opposed to focal involvement and low-density mural oedema as opposed to enhancement. Finally, the presence of metastasis, such as in the liver, would favour a malignant aetiology. There is, however, considerable overlap in the features of CRC and these inflammatory and infective conditions. Ultimately, it is ideal to perform an optical colonoscopy to confirm the diagnosis.

\section{PITFALLS OF DIAGNOSIS OF CRC ON NON-CTC ABDOMINOPELVIC CT}

Factors that hinder the detection of CRC include inadequate bowel distension, presence of faecal material, absence of IV contrast and partial volume effect of colonic haustrations on axial images.

Inadequate bowel distension is not uncommon on routine abdominopelvic CT and can reduce the sensitivity of detection of colonic tumours (Fig. 2). However, there are several imaging clues that can help differentiate between true mural thickening and under-distension or peristalsis. Pathological mural thickening in CRC usually appears irregular or nodular, shows homogenous enhancement, may be associated with pericolic abnormalities (Fig. 3) and is persistent on multiple phases, positions or historical images (Figs. $4 \& 5$ ). On the other hand, in colonic underdistension or peristalsis, mural thickening is usually smooth, shows the same degree of enhancement as the rest of the bowel, changes with phase, positions and historical images and has no pericolic abnormality.

Residual faecal material in the non-prepared and underdistended colon may obscure small mucosal lesions. However, when the colon is distended with stool, faecal material can sometimes serve as a negative bowel contrast agent when it 

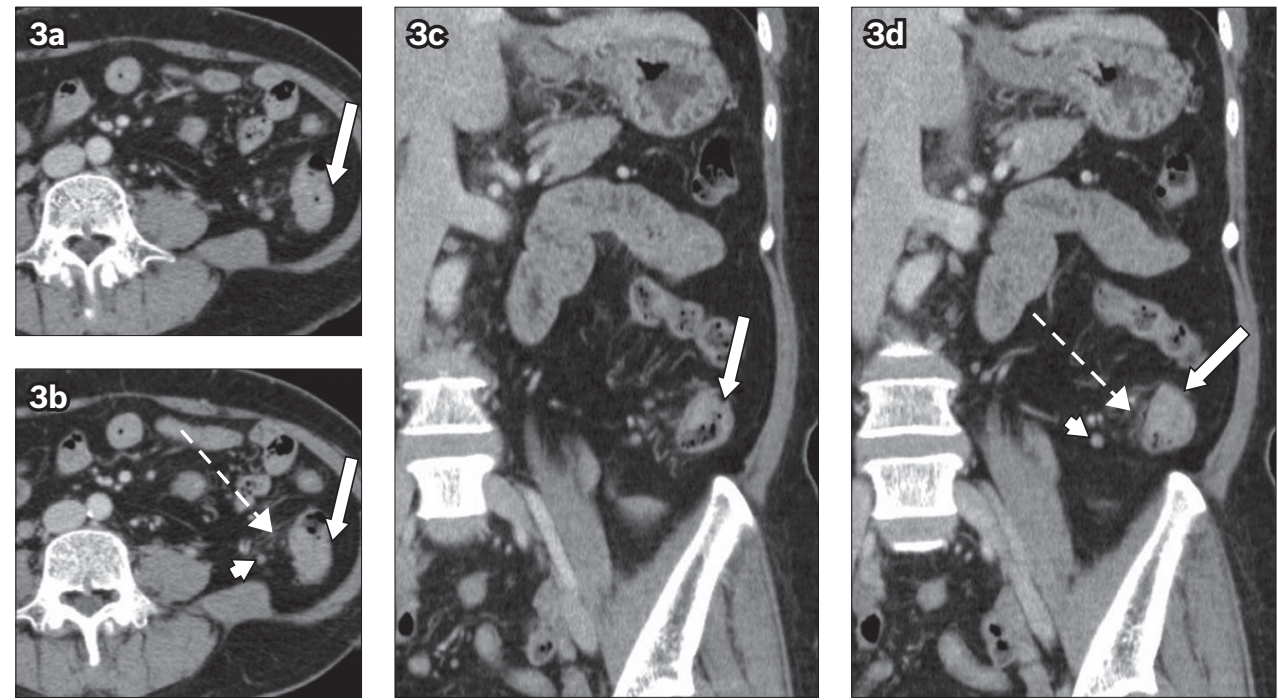

Fig. 3 A 40-year-old woman with nonspecific abdominal pain, possibly ureteric colic. (a \& b) Axial and (c \& d) coronal CT images show diffusely underdistended colon with faeces. There is suggestion of focal enhancing eccentric mural thickening in the proximal descending colon (arrow), better seen in the coronal images. Focal pericolic fat stranding (broken arrows) and adenopathy (arrowheads) are seen, which raises the suspicion of a tumour. Optical colonoscopy later confirmed adenocarcinoma.
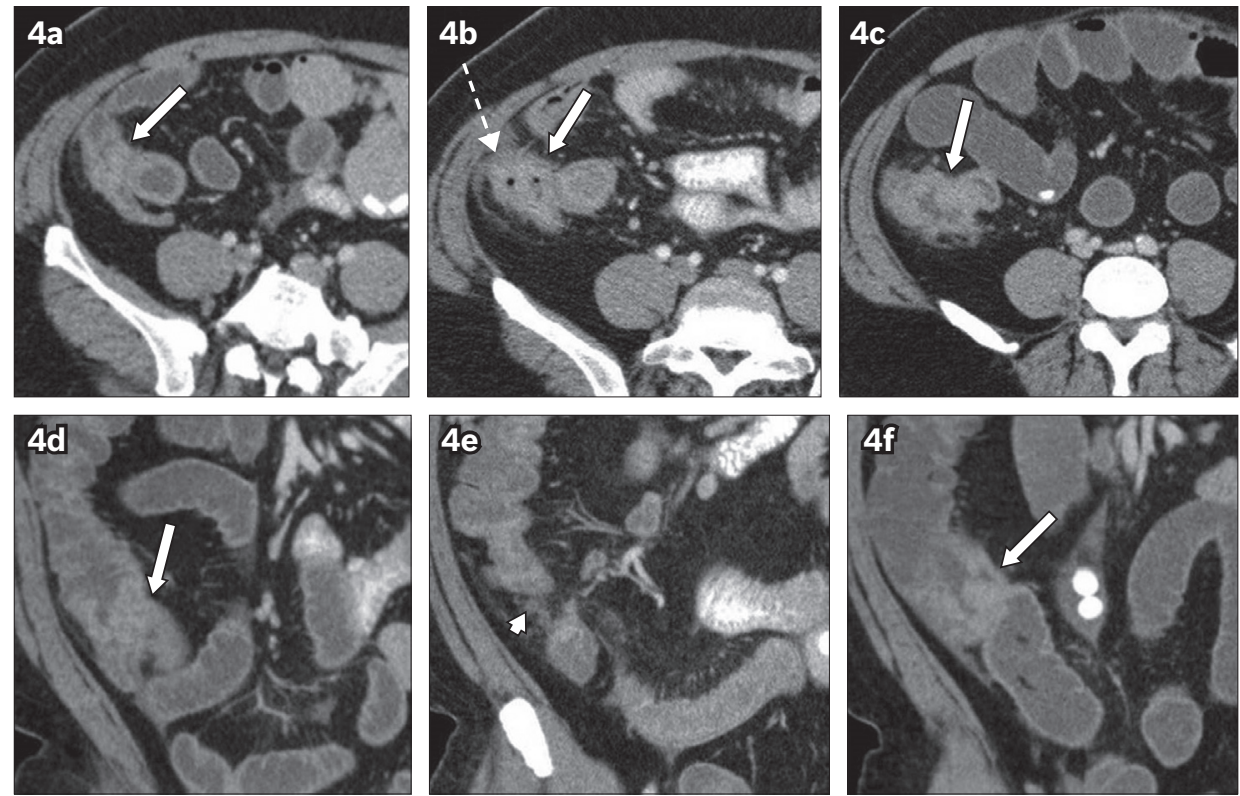

Fig. 4 A 45-year-old man presented with epigastric pain in September 2008. (a-c) Paired axial and $(d-f)$ coronal sequential CT images from September 2008 to May 2009. Thickening of the ileocaecal junction was seen in September 2008, which was thought to be due to under-distended bowel (arrows in a \& d). Follow-up CT images in December 2008 (b \& e) show increased thickening (arrow) and pericolic fat stranding (broken arrow), with enlarged necrotic lymph nodes present (arrowhead). The patient defaulted colonoscopy and re-presented in May 2009 with increased eccentric thickening in the ileocaecal junction (arrows in c \& f). Colonoscopy confirmed adenocarcinoma.
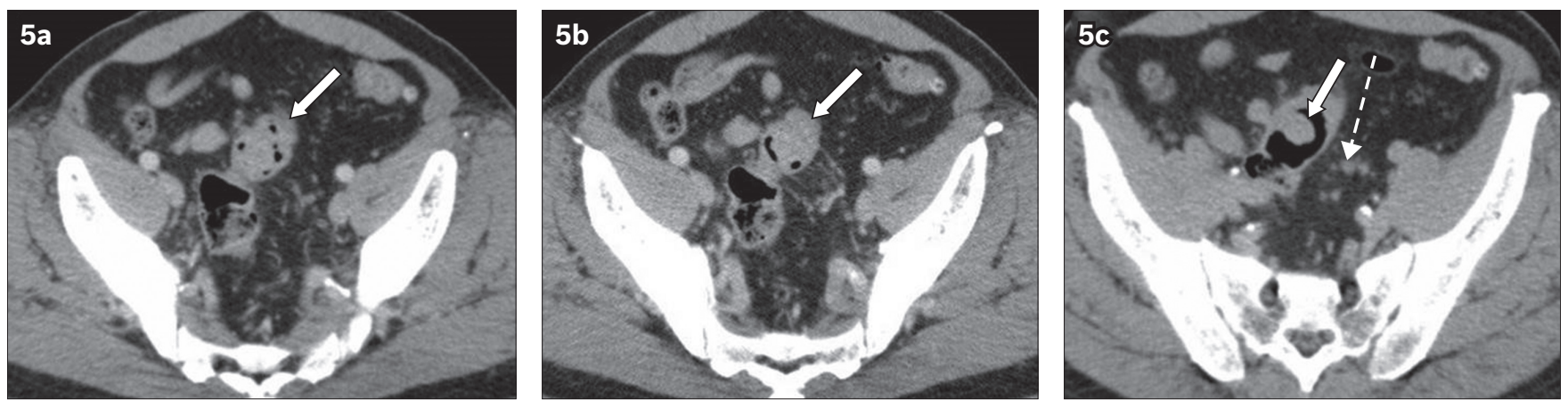

Fig. 5 CT urography was performed for a 73-year-old man who presented with haematuria and lower urinary tract symptoms in June 2011. (a \& b) Nephrographic phase axial CT images obtained in the supine position show an incidental nodular soft tissue density in an under-distended sigmoid colon (arrows). There was uncertainty as to whether it was faeces or a tumour. (c) CT image in the prone position shows air outlining a polypoidal soft tissue nodule (arrow). Small-volume focal pericolic nodes (broken arrow) were also present. Colonoscopy confirmed a 2-cm polyp and histology showed adenocarcinoma. 

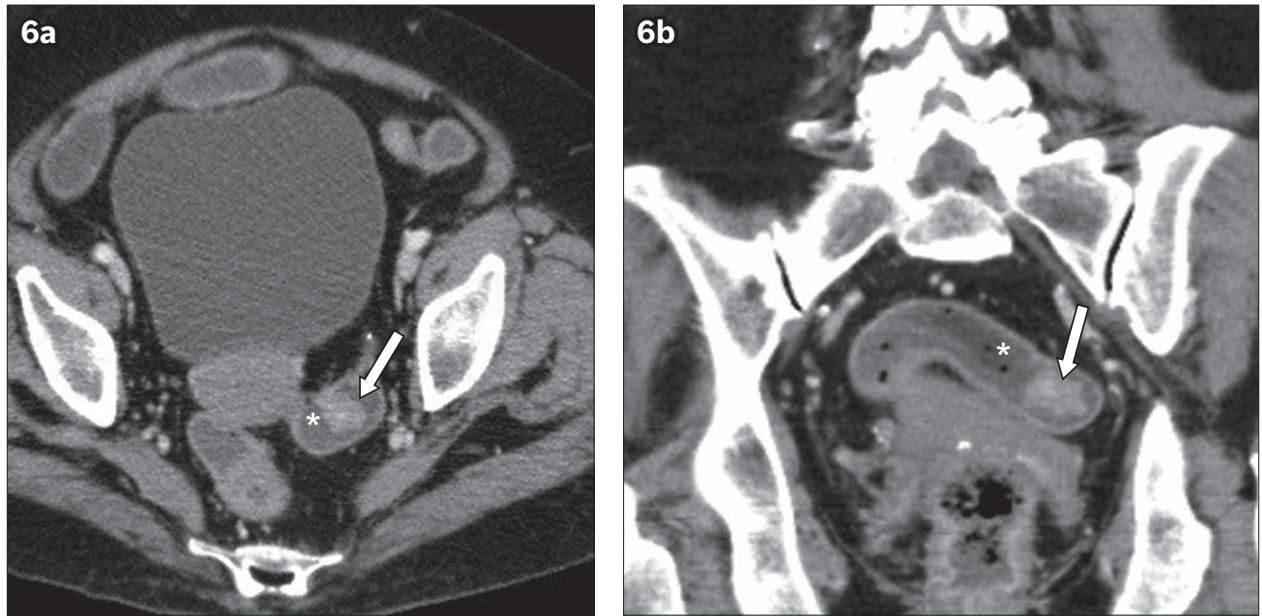

Fig. 6 A 79-year-old woman was admitted with intestinal obstruction secondary to a para-umbilical hernia in May 2009. (a) Axial and (b) coronal CT images show an incidental enhancing polypoidal lesion in the anti-dependent wall of the rectosigmoid colon (arrows), outlined by low-density liquid stool (*). This was missed in the original interpretation. The patient presented several months later with rectal bleeding. Colonoscopy confirmed a polyp in the sigmoid colon. Histology showed moderately differentiated adenocarcinoma.
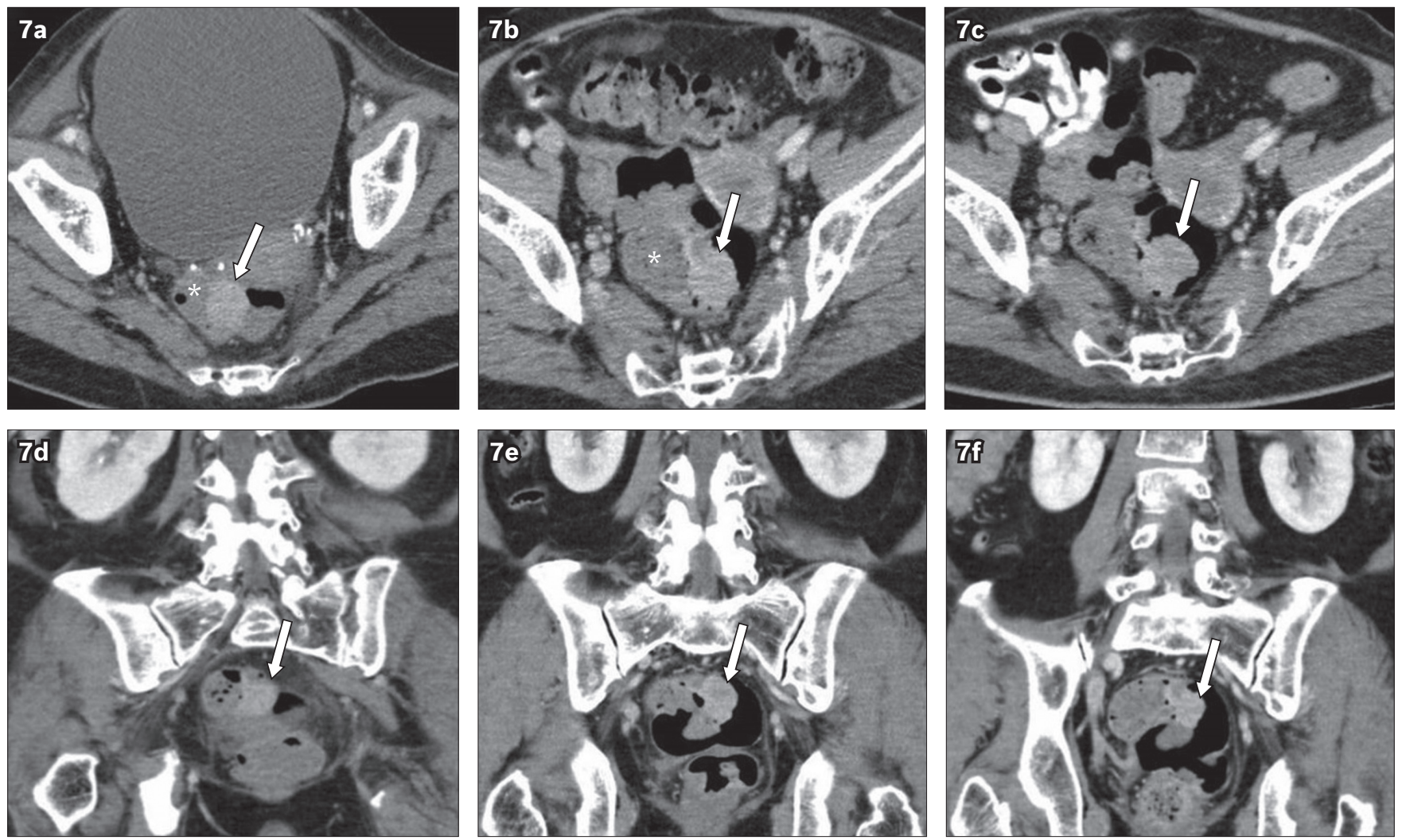

Fig. 7 A 71-year-old woman presented with a liver abscess. $(a-c)$ Axial and $(d-f)$ coronal sequential CT images performed to follow up on treatment for the abscess. There was incidental persistent polypoidal enhancing soft tissue in the sigmoid colon (arrows in a \& d) outlined by low-density faeces (*), which was not detected in the antecedent CT image in April 2009 and the follow-up CT image in June 2009 (arrows in b \& e). It was finally detected in the August 2009 CT images (arrows in c \& f). Colonoscopy and histology confirmed adenocarcinoma.

surrounds the soft tissue lesion. Solid faeces have a mottled appearance that contains gas pockets, while liquid stool has the appearance of low-attenuation contrast. In these cases, the mass may appear as a 'filling defect' (Figs. 6 \& 7).

The absence of IV contrast can make it difficult to differentiate faeces from soft tissue. Contrast agents may be omitted due to contraindications such as in cases of renal failure or contrast allergy. During CT colonography, IV contrast improves conspicuity and diagnostic confidence in polyp detection. ${ }^{(7)}$ In the absence of IV contrast, it is difficult to evaluate the colon, especially if it is under-distended on abdominopelvic CT. For such cases, secondary signs such as pericolic adenopathy, neovascularity and pericolic mesenteric fat stranding can help in CRC detection (Figs. 8 \& 9).

Another pitfall is partial volume effect of colonic haustrations on axial images, which can mimic colonic wall thickening; conversely, such tumours may be mistaken for colonic haustrations (Figs. 10 \& 11). Coronal reformatted images are often useful in distinguishing between haustrations and true colonic wall thickening. 

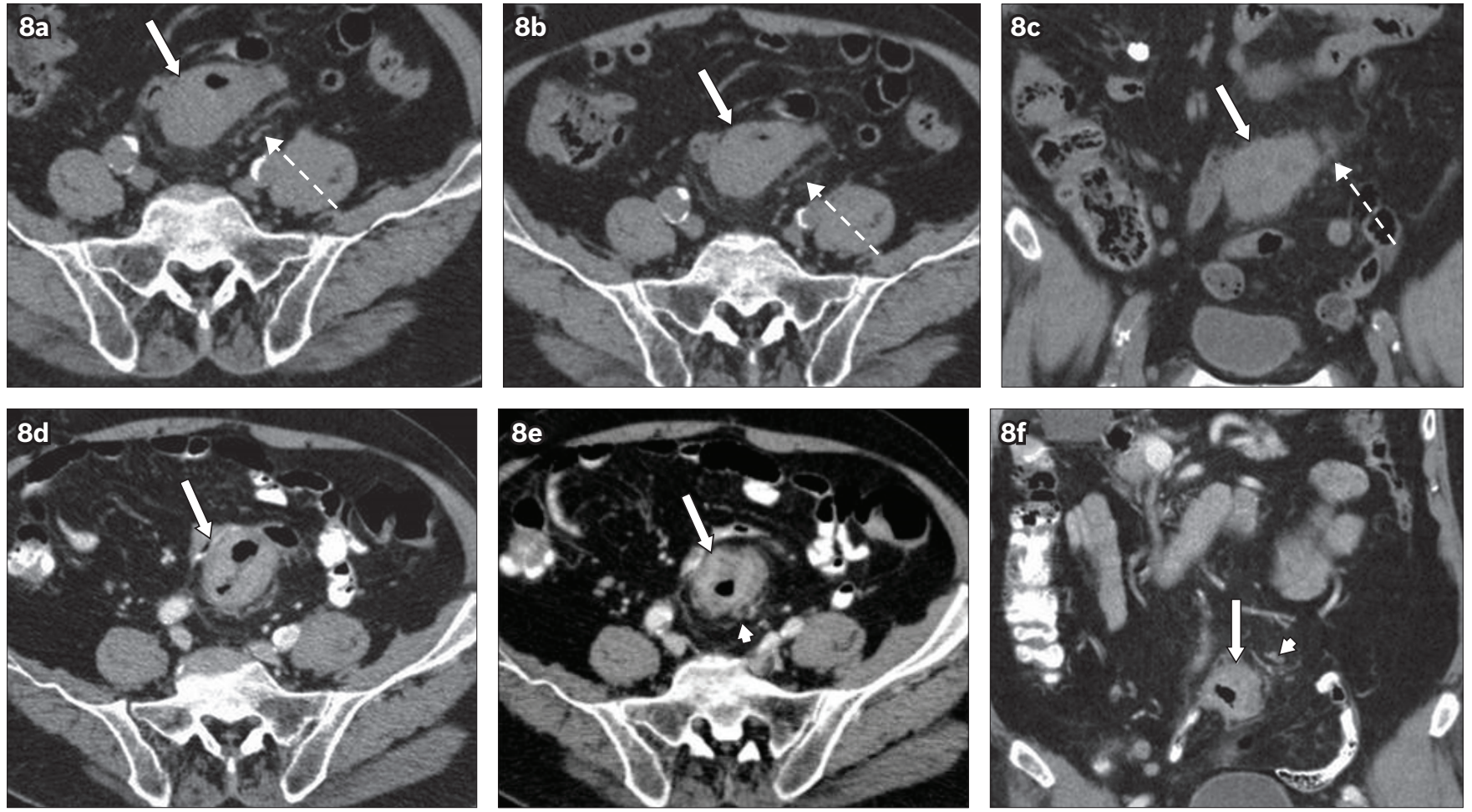

Fig. 8 An 87-year-old man presented with haematuria in November 2008. (a-c) Unenhanced CT KUB (kidney, ureter, bladder) images show no urinary stones. There was incidental circumferential mural thickening of the sigmoid colon (arrows) with subtle pericolic fat stranding (broken arrows), which was not detected in the original interpretation. The patient re-presented in January 2009 with rectal bleeding. (d-f) Contrast-enhanced CT images of the abdomen and pelvis clearly show an enhancing sigmoid mass (arrows) with pericolic fat stranding and small lymph nodes (arrowheads). Colonoscopy confirmed adenocarcinoma.
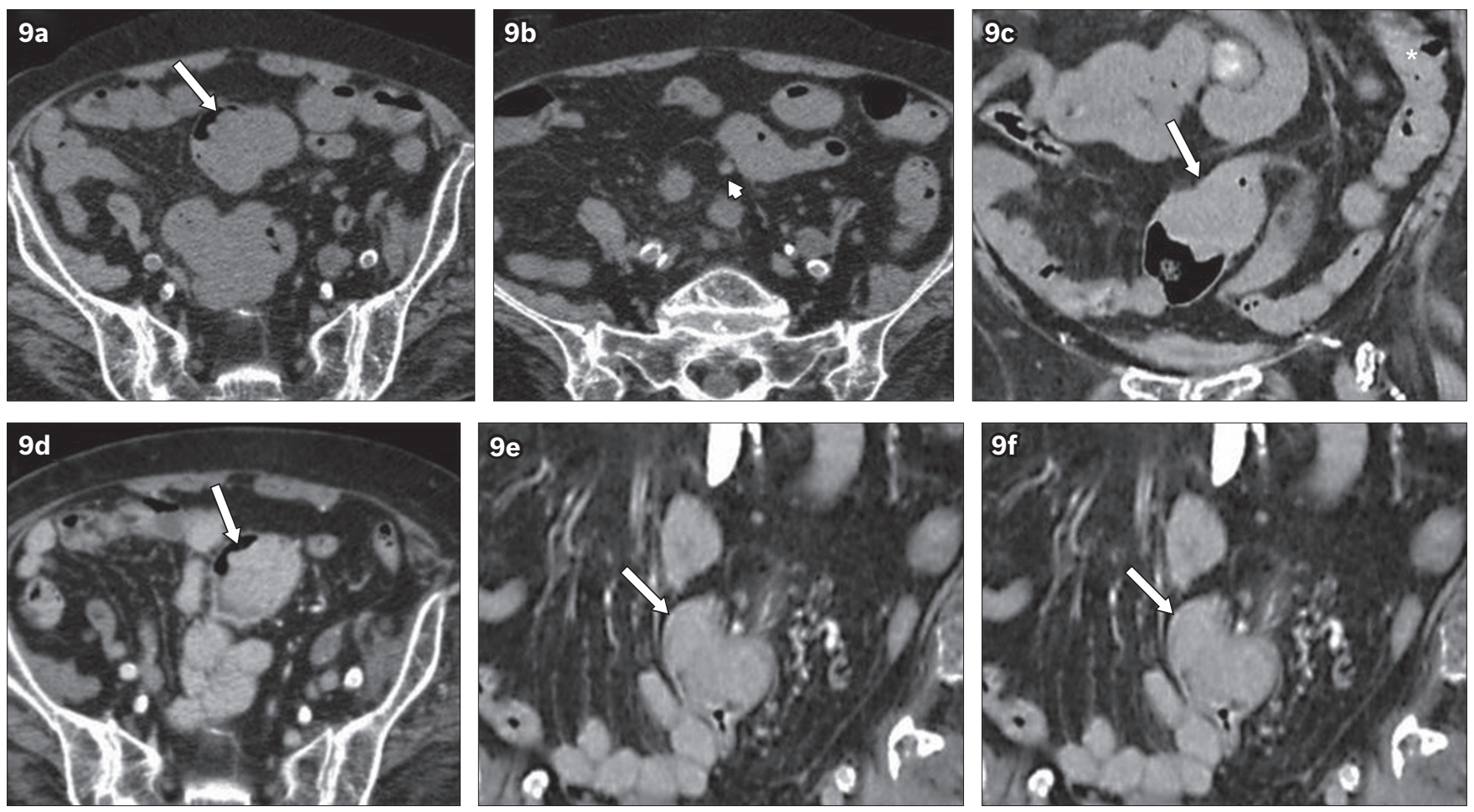

Fig. 9 An 87-year-old woman presented in August 2011 with a distal left ureteric stone causing hydronephrosis. (a \& b) Unenhanced axial and (c) coronal CT images show an intraluminal soft tissue lesion in the sigmoid colon (arrows), which was originally interpreted as faeces. Note the identical attenuation of faeces in the descending colon ( ${ }^{*}$ in c). There was a small pericolic node (arrowhead in b). The patient re-presented in November 2011 with rectal bleeding. (d-f) CT images clearly show an enhancing mass distending the sigmoid colon (arrows). Histology showed adenocarcinoma.

\section{COMPLICATIONS OF CRC AS FIRST PRESENTATION}

Some patients with CRC present with complications such as bowel obstruction and perforation, abscesses, acute appendicitis or intussusception instead of primary effects of the tumour such as gastrointestinal bleeding, tenesmus or constipation. ${ }^{(8,15)}$ In these patients, it is important to evaluate for underlying CRC, which may be masked by the acute findings. 

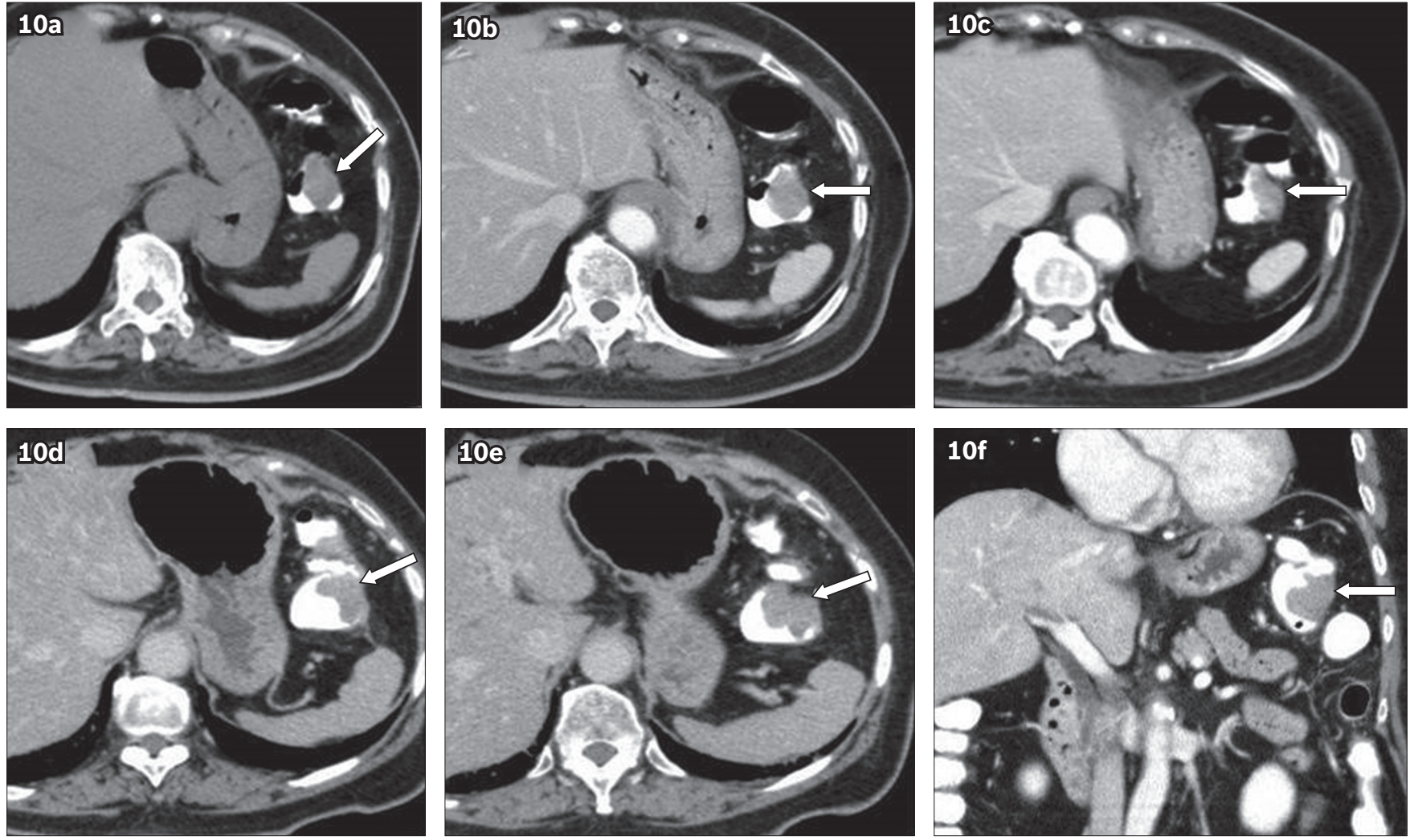

Fig. 10 A 76-year-old woman presented with left iliac fossa pain in December 2006. (a-c) Axial contrast-enhanced CT images of the abdomen and pelvis show eccentric mucosal thickening at the splenic flexure of the colon (arrows), which was outlined by bowel contrast. This was mistaken for partial volume effect of haustration, as no coronal images were available at the time. The patient re-presented in March 2008 with rectal bleeding. (d \& e) CT images show a mass in the splenic flexure (arrows). (f) Coronal reformatted image confirmed a mass (arrow). Colonoscopy confirmed adenocarcinoma.
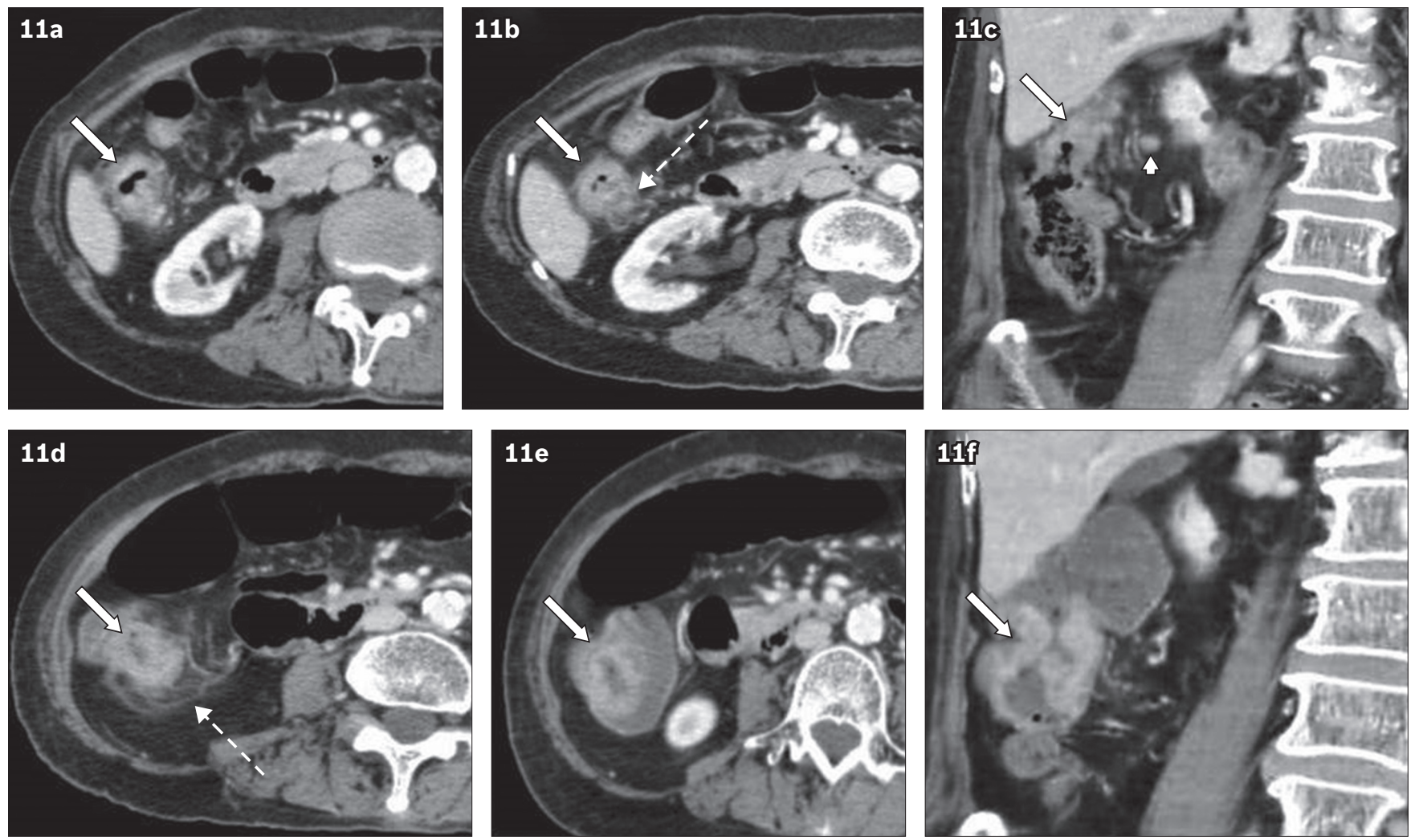

Fig. 11 An 80-year-old woman presented with nocturia and frequency in April 2009. (a \& b) Axial and (c) coronal CT urogram images (in the nephrographic phase) show subtle enhancing mural thickening in the hepatic flexure (arrows) with pericolic fat stranding (broken arrow in b) and a lymph node (arrowhead in c) in a poorly distended colon. This was originally thought to be partial volume effect of colonic haustration. The patient re-presented in July 2009 with rectal bleeding. (d \& e) CT images show eccentric wall thickening (arrows) and pericolic fat stranding (broken arrow). (f) Coronal image clearly shows an 'apple-core' tumour (arrow), which was confirmed on histology. 

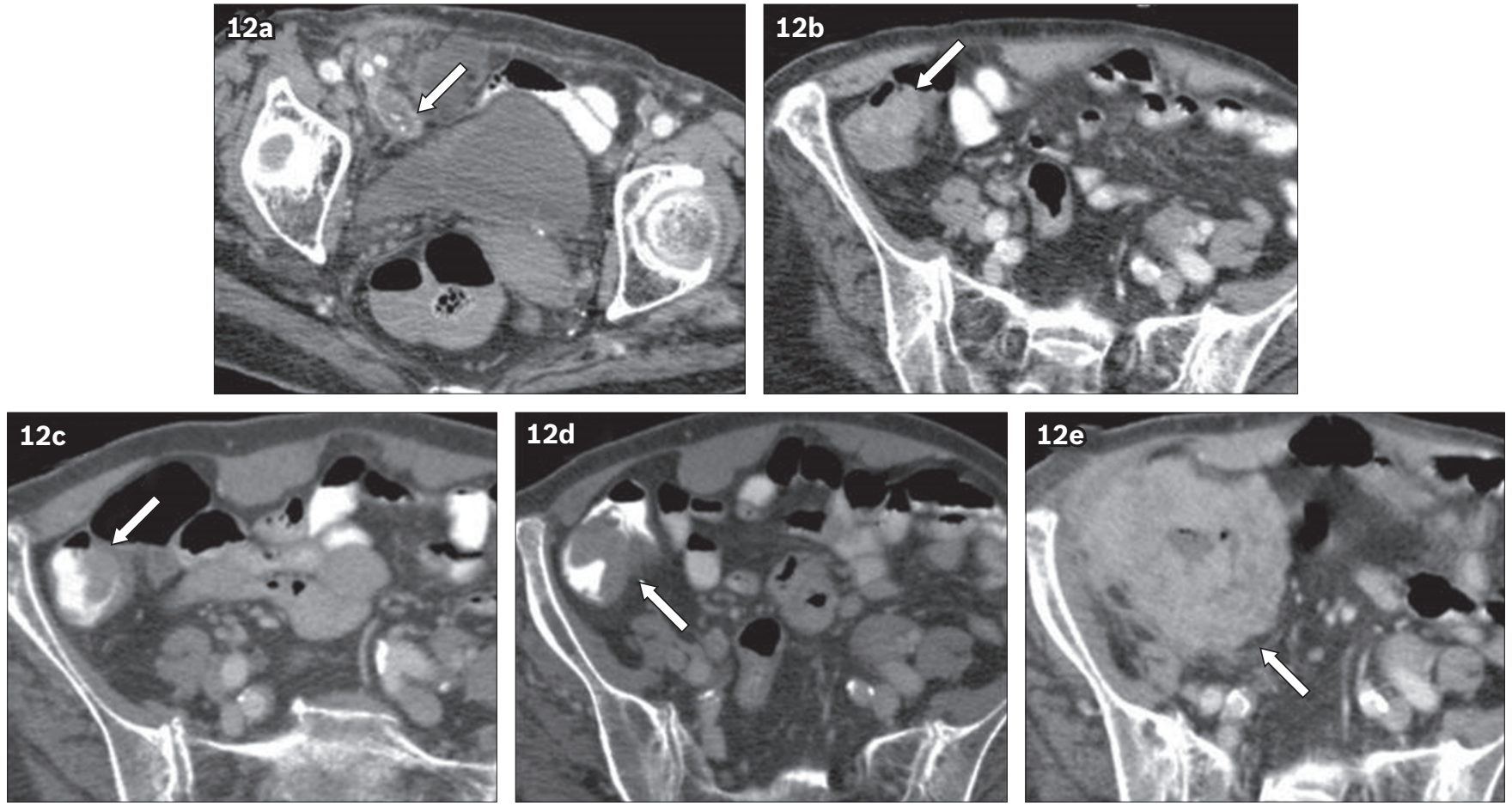

Fig. 12 A 78-year-old woman presented with right iliac fossa pain. Axial CT images show (a) a dilated, fluid-filled appendix with appendicoliths (arrow), consistent with acute appendicitis; (b) thickening and under-distension of the proximal ascending colon (arrow), thought to be related to appendiceal inflammation. The appendicitis was ascribed to obstruction from appendicoliths. The patient was treated conservatively due to comorbidities. (c) Followup axial CT image shows resolution of the right iliac fossa inflammation and eccentric thickening of the medial wall of the proximal ascending colon (arrow). (d) CT image, taken six months later, shows increased thickening (arrow). In both (c) and (d), the thickening was interpreted as under-distension, peristalsis or partial volume effect of a haustral fold. (e) CT image confirms that the frank malignant mass in the proximal ascending colon (arrow) was adenocarcinoma.

Bowel obstruction is the most common complication of colon cancer, with a $1 \%-10 \%$ incidence of perforation reported. ${ }^{(8,15)}$ Identifying the transition point as focal asymmetric irregular wall thickening or the presence of a mass representing CRC is crucial to establishing the cause of perforation, as perforation is associated with a higher chance of metastasis. ${ }^{(15)}$ Perforation can occur secondary to tumour necrosis or from a closed-loop obstruction in which there is raised intracolonic pressure proximal to the tumour and a competent ileocaecal valve. ${ }^{(8,15)}$ Common sites of perforation are the sigmoid colon and caecum, and the rate of perforation in the left hemicolon is higher than that in the right due to the smaller diameter. ${ }^{(15)}$

CRC that is associated with local abscess formation is rare, with a reported incidence rate of only $0.3 \%-4 \%{ }^{(8)}$ Abscess formation results from colonic perforation, fistula formation or direct invasion into the adjacent structures. The peritoneal and pelvic cavities are common locations of CRC-related abscess formation, although abscesses can also be seen in the retroperitoneum, abdominal wall, perirectal space, psoas muscle and thigh. ${ }^{\left({ }^{8}\right)}$ Pericolic fat stranding is often seen around the abscess, and thus, careful assessment of bowel wall enhancement pattern and thickening as well as the length of bowel involvement is important in differentiating between a benign inflammatory condition and CRC.

CRC can also present as acute appendicitis resulting from obstruction of the appendix by caecal cancer. This scenario is seen especially in elderly patients ${ }^{(8)}$ and failure to identify caecal cancer may lead to incomplete treatment (Fig. 12). Again, a high index of suspicion in the appropriate patient demographic and evaluation of the pattern of caecal wall enhancement and thickening are useful in differentiating between primary and secondary acute appendicitis.

Adult intussusception is rare and mostly caused by lead points such as CRC, enlarged lymph nodes or metastatic lesions. The most common lead mass for adult intussusception is a malignant lesion. CT typically shows the 'target' sign, depicting the intussusception with an enhancing lead mass (Fig. 13). One study reported that intussusceptions with a lead point are generally longer and larger in diameter compared with intussusceptions without a lead point. ${ }^{(8)}$

\section{ASSOCIATION OF CRC WITH LIVER ABSCESS}

Previously, liver abscess was known to be related to perforation of acute appendicitis and diseases of the biliary tract. Recently, multiple case reports have shown that liver abscess is also associated with various colonic diseases such as colonic diverticulitis, inflammatory bowel disease and even CRC (Fig. 14). From 1960 to 2011, a total of 96 documented cases of CRCrelated cryptogenic liver abscess were reported worldwide and in the Eastern Asian countries; the numbers have increased by 2-4 times in the past few decades. ${ }^{(16)}$ The male gender is more often affected and the mean age of patients is 64 years. ${ }^{(16)} \mathrm{A}$ high percentage of these patients have diabetes mellitus and Klebsiella pneumoniae (K. pneumoniae) of colonic origin. ${ }^{(16,17)}$ The most commonly involved segment is the sigmoid colon, followed by 

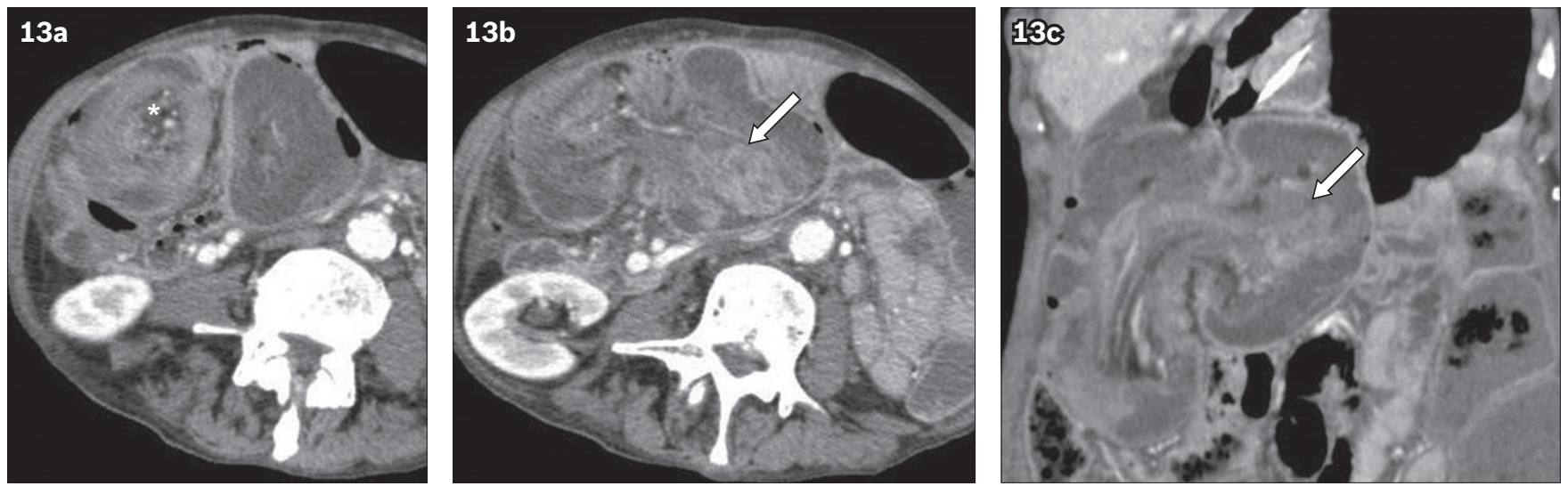

Fig. 13 A 68-year-old patient presented with large bowel obstruction. (a-c) CT images show a 'target sign' (* in a), consistent with intussusception. The intussusception was deemed to be secondary to a mass in the caecum (arrows), which was confirmed to be caecal carcinoma.
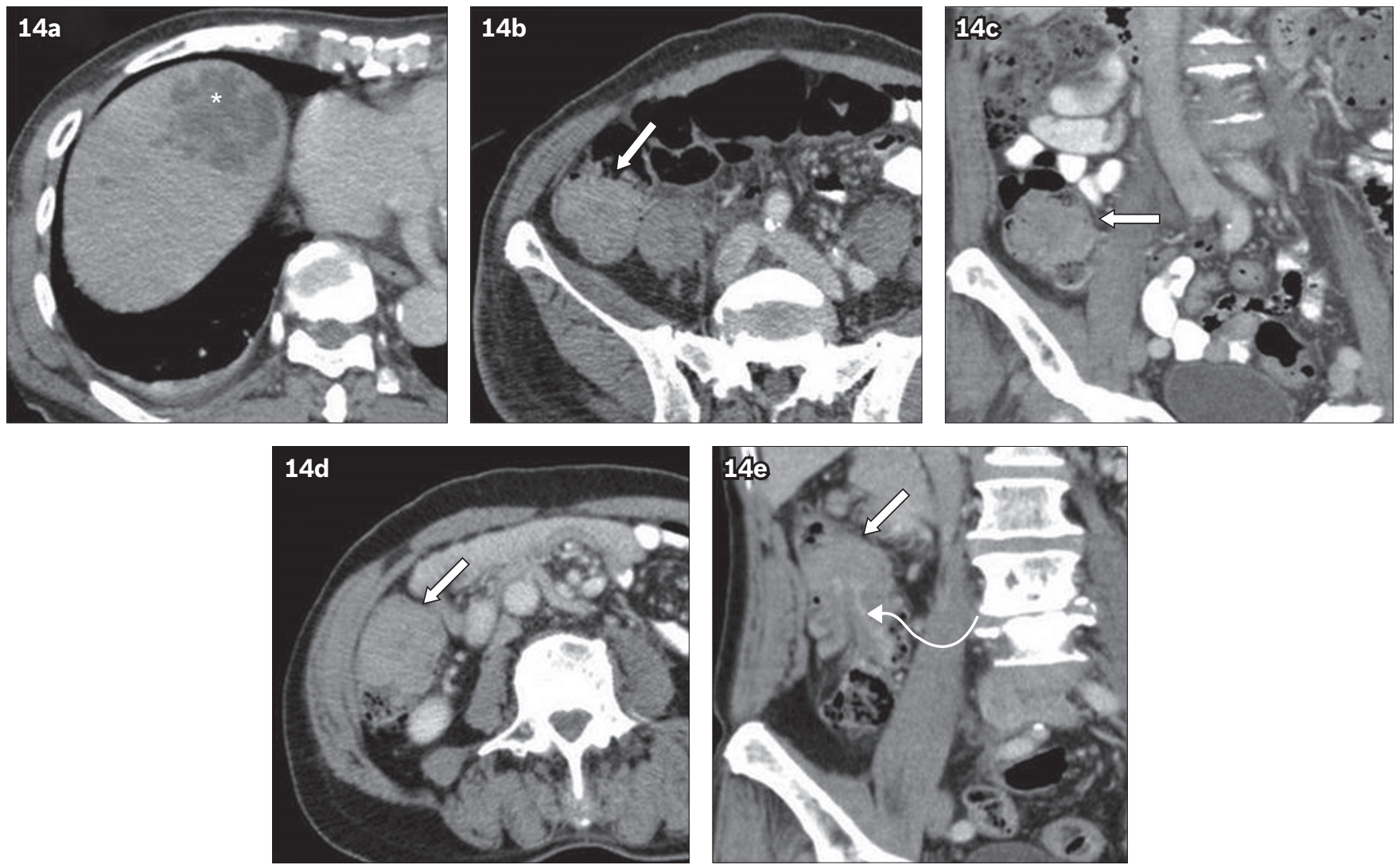

Fig. 14 A 77-year-old man presented with sepsis in March 2010. (a \& b) Axial and (c) coronal CT images show a liver abscess ( ${ }^{*}$ in a) and a soft tissue lesion in the caecum (arrows), which was not detected initially. The patient returned in May 2010 for follow-up CT for the treated abscess. (d \& e) CT images again show a persistent soft tissue lesion in the caecum (arrows) with an enhancing stalk (curved arrow in e). Colonoscopy revealed a tubulovillous adenoma with a focus of adenocarcinoma.

the rectum. Destruction of colonic mucosal barrier and bacterial translocation into the portal system with haematogenous spread to the liver have been postulated. ${ }^{(16)}$ In Jeong et al's study conducted in South Korea, among 37 patients with cryptogenic liver abscess who underwent optical colonoscopy between 2003 and 2010, $8(22 \%)$ patients had colon cancer. ${ }^{(17)}$ It is therefore important to screen for CRC in patients with cryptogenic liver abscess, especially in diabetics and those with K. pneumoniae.

\section{CONCLUSION}

CRC is a common cancer and patients are often asymptomatic at an early stage of the disease. As abdominopelvic CT is increasingly performed for general abdominal and urinary symptoms, the radiologist may be the first person to suggest a diagnosis of CRC. This article reviews the radiologic findings, pitfalls and limitations as well as uncommon presentations of complicated CRC on abdominopelvic CT. Knowledge of these findings will improve the accuracy of a diagnosis of CRC on routine abdominopelvic CT using optimal evaluation methods.

\section{REFERENCES}

1. American Cancer Society. Colorectal Cancer Facts \& Figures 2014-2016. Available at: http://www.cancer.org/acs/groups/content/documents/ document/acspc-042280.pdf. Accessed May 3, 2014.

2. Haggar FA, Boushey RP. Colorectal cancer epidemiology: incidence, 
mortality, survival, and risk factors. Clin Colon Rectal Surg 2009; 22:191-7.

3. Jemal A, Bray F, Center MM, et al. Global cancer statistics. CA Cancer J Clin 2011; 61:69-90.

4. Yee J, Weinstein S, Morgan T, Alore P, Aslam R. Advances in CT Colonography for Colorectal Cancer Screening and Diagnosis. J Cancer 2013; 4:200-9.

5. Atkin WS, Saunders BP; British Society for Gastroenterology; Association of Coloproctology for Great Britain and Ireland. Surveillance guidelines after removal of colorectal adenomatous polyps. Gut 2002; 51 Suppl 5:V6-9.

6. Pickhardt PJ, Choi JR, Hwang I, et al. Computed tomographic virtua colonoscopy to screen for colorectal neoplasia in asymptomatic adults. N Engl J Med 2003; 349:2191-200.

7. Silva AC, Hara AK, Leighton JA, Heppell JP. CT colonography with intravenous contrast material: varied appearances of colorectal carcinoma. Radiographics 2005; 25:1321-34.

8. Kim SW, Shin HC, Kim IY, Kim YT, Kim CJ. CT findings of colonic complications associated with colon cancer. Korean J Radiol 2010 11:211-21

9. Horton KM, Abrams RA, Fishman EK. Spiral CT of colon cancer: imaging features and role in management. Radiographics 2000; 20:419-30.

10. Xiong L, Chintapalli KN, Dodd GD 3rd, et al. Frequency and CT patterns of bowel wall thickening proximal to cancer of the colon. AJR Am J Roentgenol 2004; 182:905-9.

11. Chintapalli KN, Chopra S, Ghiatas AA, et al. Diverticulitis versus colon cancer: differentiation with helical CT findings. Radiology 1999; 210:429-35.

12. Padidar AM, Jeffrey RB Jr, Mindelzun RE, Dolph JF. Differentiating sigmoid diverticulitis from carcinoma on $\mathrm{CT}$ scans: mesenteric inflammation suggests diverticulitis. AJR Am J Roentgenol 1994; 163:81-3.

13. Macari M, Balthazar EJ. CT of bowel wall thickening: significance and pitfalls of interpretation. AJR Am J Roentgenol 2000; 176:1105-16.

14. Chintapalli KN, Esola CC, Chopra S, Ghiatas AA, Dodd GD 3rd. Pericolic mesenteric lymph nodes: an aid in distinguishing diverticulitis from cancer of the colon. AJR Am J Roentgenol 1997; 169:1253-5.

15. Kim SW, Kim HC, Yang DM. Perforated tumours in the gastrointestinal tract: CT findings and clinical implications. $\mathrm{Br}$ J Radiol 2012; 85:1307-13

16. Qu K, Liu C, Wang ZX, et al. Pyogenic liver abscesses associated with nonmetastastic colorectal cancers: An increasing problem in Eastern Asia. World J Gastroenterol 2012; 18:2948-55.

17. Jeong SW, Jang JY, Lee TH, et al. Cryptogenic pyogenic liver abscess as the herald of colon cancer. J Gastroenterol Hepatol 2012; 27:248-55. 


\section{SINGAPORE MEDICAL COUNCIL CATEGORY 3B CME PROGRAMME} (Code SMJ 201505A)

Question 1. Regarding the epidemiology and aetiology of colorectal carcinoma (CRC):

(a) The incidence and mortality rates for CRC increase with age, with a majority of cases seen in patients 50 years or older.

(b) The adenoma-carcinoma sequence has a natural history of five years.

(c) Most patients with early CRC are symptomatic.

(d) The most common symptom in patients with CRC is rectal bleeding.

Question 2. Regarding computed tomography colonography (CTC) and non-CTC abdominopelvic CT:

(a) CTC is comparable to optical colonoscopy in CRC detection.

(b) Bowel preparation, faecal tagging and colonic distension are not part of the non-CTC abdominopelvic protocol.

(c) Lack of bowel preparation, residual faecal material and colonic under-distension decrease the sensitivity of non-CTC abdominopelvic CT in the detection of CRC.

(d) The patient is usually scanned only in the supine position on non-CTC abdominopelvic CT.

Question 3. Regarding non-CTC abdominopelvic CT imaging in CRC:

(a) The typical appearance of CRC is short segment eccentric bowel wall thickening and enhancement.

(b) Focal pericolic neovascularity, fat stranding and locoregional adenopathy, although nonspecific, may represent secondary signs of CRC.

(c) The lung is the most common site of metastasis for CRC.

(d) Imaging findings of inflammatory bowel diseases may mimic CRC.

Question 4. Regarding pitfalls in diagnosis of CRC on non-CTC abdominopelvic CT:

(a) Colonic under-distension can be mistaken for mural thickening in CRC.

(b) Residual faecal material may obscure small polyps or masses.

(c) Partial volume effect of colonic haustrations, which may mimic colonic wall thickening on axial images, can be resolved by reformatting in another plane (such as coronal).

(d) Historical studies are unhelpful in determining the presence of CRC.

Question 5. Regarding complications and associations of CRC:

(a) Large bowel obstruction is a common complication of CRC.

(b) CRC presenting as acute appendicitis due to obstruction of the appendix by caecal cancer is usually seen in middle-age patients.

(c) CRC can cause intussusception in adults.

(d) There is no known association between CRC and cryptogenic liver abscess.

True

False

$\square$

$\square$

\footnotetext{
Doctor's particulars:

Name in full

MCR number

Email address

Specialty:

SUBMISSION INSTRUCTIONS:

(1) Log on at the SMJ website: http://www.sma.org.sg/publications/smjcurrentissue.aspx and select the appropriate set of questions. (2) Provide your name, email address and MCR number. (3) Select your answers and click "Submit".

\section{RESULTS:}

(1) Answers will be published in the SMJ July 2015 issue. (2) The MCR numbers of successful candidates will be posted online at the SMJ website by 29 June 2015. (3) Passing mark is $60 \%$. No mark will be deducted for incorrect answers. (4) The SMJ editorial office will submit the list of successful candidates to the Singapore Medical Council. (5) One CME point is awarded for successful candidates.

Deadline for submission: (May 2015 SMJ 3B CME programme): 12 noon, 22 June 2015.
} 